\title{
Pathogenesis of Focal Segmental Glomerular Sclerosis in a Girl with the Partial Deletion of Chromosome 6p
}

\author{
Akane Izu, ${ }^{1}$ Hidehiko Yanagida, ${ }^{1}$ Keisuke Sugimoto, ${ }^{1}$ Shinsuke Fujita, ${ }^{1}$ \\ Naoki Sakata, ${ }^{1}$ Norihisa Wada, ${ }^{1}$ Mitsuru Okada ${ }^{1}$ and Tsukasa Takemura ${ }^{1}$ \\ ${ }^{1}$ Department of Pediatrics, Kinki University School of Medicine, Osaka, Japan
}

\begin{abstract}
Focal segmental glomerular sclerosis (FSGS) is a leading cause of the nephrotic syndrome and characterized by the sclerosing lesions that affect one or more segments of some glomeruli. We encountered a female patient with a partial deletion of chromosome $6 p$, who presented proteinuria at age 3 years. Detailed chromosomal analysis disclosed an interstitial deletion of $6 \mathrm{p}$ : del(6)(p22.1p22.3). No abnormality such as hydronephrosis or renal agenesis was disclosed by imaging, but FSGS was present in a renal biopsy specimen. The patient is currently 11 years old and shows mental retardation with mild deterioration in the renal function. To address the defective genes in the present patient, we carried out comparative genomic hybridization $(\mathrm{CGH})$, showing that $E 2 F 3$ on chromosome $6 p$ is absent in this patient. $\mathrm{E} 2 \mathrm{~F} 3$, a member of the E2F family transcription factors, inhibits expression of vascular endothelial growth factor (VEGF) and induces apoptosis during vascular development. The deletion of E2F3 was also detected by employing a PCR method, suggesting that glomerular architecture had been compromised in this patient. Serum VEGF concentrations were elevated to $177 \pm 21.4 \mathrm{pg} / \mathrm{mL}$ (upper limit of $33.3 \mathrm{pg} / \mathrm{mL}$ ), when she was 6 years old, associated with the enhanced expression of VEGF in glomeruli. These findings suggest that the dysregulation of VEGF synthesis caused by the deletion of E2F3 may be associated with development of FSGS. In conclusion, among patients with idiopathic FSGS, an abnormality of E2F3 may exist on chromosome $6 \mathrm{p}$. Therefore, one might consider chromosomal analyses in children with FSGS who have mental retardation.
\end{abstract}

Keywords: apoptosis; comparative genomic hybridization; $E 2 F 3$ gene; transcription factor; vascular endothelial growth factor

Tohoku J. Exp. Med., 2011, 223 (3), 187-192. C 2011 Tohoku University Medical Press

Only about 30 occurrences of the partial deletion of chromosome $6 \mathrm{p}$, a very rare chromosomal aberration, have been reported (Palmer et al. 1991). Most of these patients had one or more deletions in the region of $6 \mathrm{p} 22-6 \mathrm{p} 25$ (Palmer et al. 1991; Davies et al. 1996, 1999). Such deletion may present with various external anomalies including syndactyly, brevicollis, microcephaly, microphthalmos, ocular hypertelorism, saddle nose, elongated philtrum, tentlike lips, low-set ears, and limb abnormalities; viscerally, cardiac and renal anomalies may occur. Renal complications reported to date include hydronephrosis caused by stenosis of the ureteropelvic junction, cystic kidney, and other defects (Davies et al. 1999).

Focal segmental glomerular sclerosis (FSGS) is characterized by the occurrence of sclerosing lesions, often with hyaline inclusions, affecting one or more segments of some but not all glomeruli (Boyer et al. 2007). This condition commonly progresses toward chronic renal failure, although the speed with which this occurs varies considerably from case to case (Boyer et al. 2007). The mutations in the Wilm's tumor 1 (WT1) or $\alpha$-actinin 4 genes are responsible for some cases of FSGS; however, there are patients with FSGS who show no abnormality in these genes (D'Agati 2008). Thus, hitherto undetermined genes might be involved in the pathogenesis of FSGS. In addition, cases of FSGS in mentally retarded children have also been reported (Joh et al. 1991; Sartelet et al. 2008).

We previously reported a present girl with the interstitial deletion of chromosome $6 p$ showing FSGS described here (Izu et al. 2011), but the precise pathogenesis of complicating FSGS remained unclear. In the present study, we investigated the pathogenesis of FSGS in this patient.

\footnotetext{
Polymerase chain reaction (PCR)

Genomic DNA was extracted from leukocytes of the present patient and a healthy individual. Genomic DNA was recovered in the aqueous phase and precipitated with ethanol/ sodium acetate. PCR
}

Methods

Received December 28, 2010; revision accepted for publication February 3, 2011. doi: 10.1620/tjem.223.187

Crrespondence: Tsukasa Takemura, M.D., Ph.D., Department of Pediatrics, Kinki University School of Medicine, 377-2 Ohno-higashi, Osaka-Sayama 589-8511, Japan. e-mail: tsukasa@med.kindai.ac.jp 
was carried out as described previously (Feber et al. 2004). Specific primers were constructed based on previously published sequence data for human E2F3 (Feber et al. 2004). After amplification, reaction products were analyzed directly using a capillary sequencer (MegaBACE1000, Amersham Bioscience, Piscataway, NJ, USA).

\section{Array Comparative Genomic Hybridization (CGH)}

We obtained genomic DNA from a reference $(46, \mathrm{XX})$ (Promega $\mathrm{p} / \mathrm{n}$ G1521) and the present patient. Array CGH was performed using premade Agilent oligo-CGH arrays (244 k arrays; Agilent Technologies, Palo Alto, CA, USA) consisting of 244,000 in situ synthesized 60-mer oligonucleotides spanning the entire genome, resulting in an average genomic distance of $\sim 12 \mathrm{~kb}$. These probes included both coding and non-coding sequences on every human chromosome. Hybridization was carried out according to the manufacture's instruction, and data were visualized by means of the

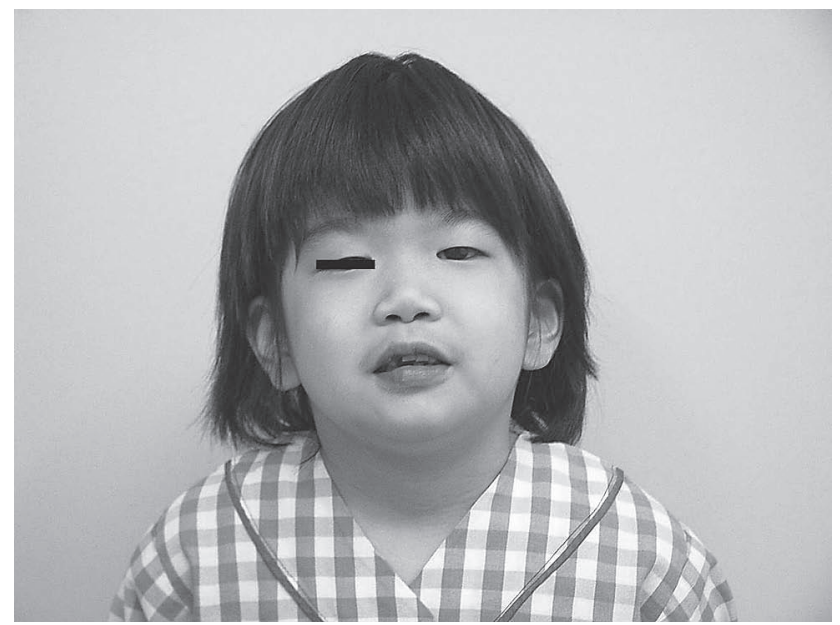

Fig. 1. Appearance of the patient. A panel shows microphthalmia, ocular hypertelorism, saddle nose, an elongated philtrum, tent-like lips, and low-set ears. Note that we obtained the approval granted to use a photograph imaging patient's figure from her parents.
CGHAnalytics 3.4 software (Agilent Technologies).

Immunohistochemical staining

Anti-vascular endothelial growth factor (VEGF) antibody was purchased from Santa Cruz Biotechnology (Santa Cruz, CA). Staining for these molecules in the patient's renal tissue was carried out using an immunoperoxidase method previously described (Takemura et al. 2010).

\section{Clinical Report}

The patient's physical findings and clinical course were reported previously (Izu et al. 2011 in press). Briefly, short stature $(-2.0 \mathrm{SD})$ and mild mental developmental retardation were evident at a health checkup at 3 years of age, proteinuria also was detected at this time. She has saddle nose, elongated philtrum, low-set ears, and tent-like lips representing the characteristic features of $6 \mathrm{p}$ partial deletion syndrome (Fig. 1). In chromosomal analysis performed after obtaining informed consent from the parents, a partial short arm deletion was detected on chromosome 6, specifically the interstitial deletion del(6)(p22.1p22.3) (Fig. 2). The patient is currently 11 years old. The girl's parents were not consanguineous, and the family history included no similar symptoms and no terminal renal failure. The patient has bronchial asthma and shows periodical attack of the disease from early childhood; thus, she is given fluticasone inhalation at present.

Using oligo-comparative genomic hybridization arrays (244 k arrays; Agilent Technologies, Palo Alto, CA), CGH was performed. Almost all genes normally present in the region were absent (Fig. 3a); deleted identified genes included RBM24, CAP2, FAM8A1, NUP153, KIF13A, NHLRC1, TPMT, AOF1, DEK, IBRDC2, ID4, SOX4, CDKAL1, E2F3, PRL, HDGFL1, NRSN1, DCDC2, KAAG1, MRS2L, GPLD1, GPLD1, ALDH5A1, KIAA0319, TTRAP, THEM2, C6orf62, and GMNN. Among these, only an E2F

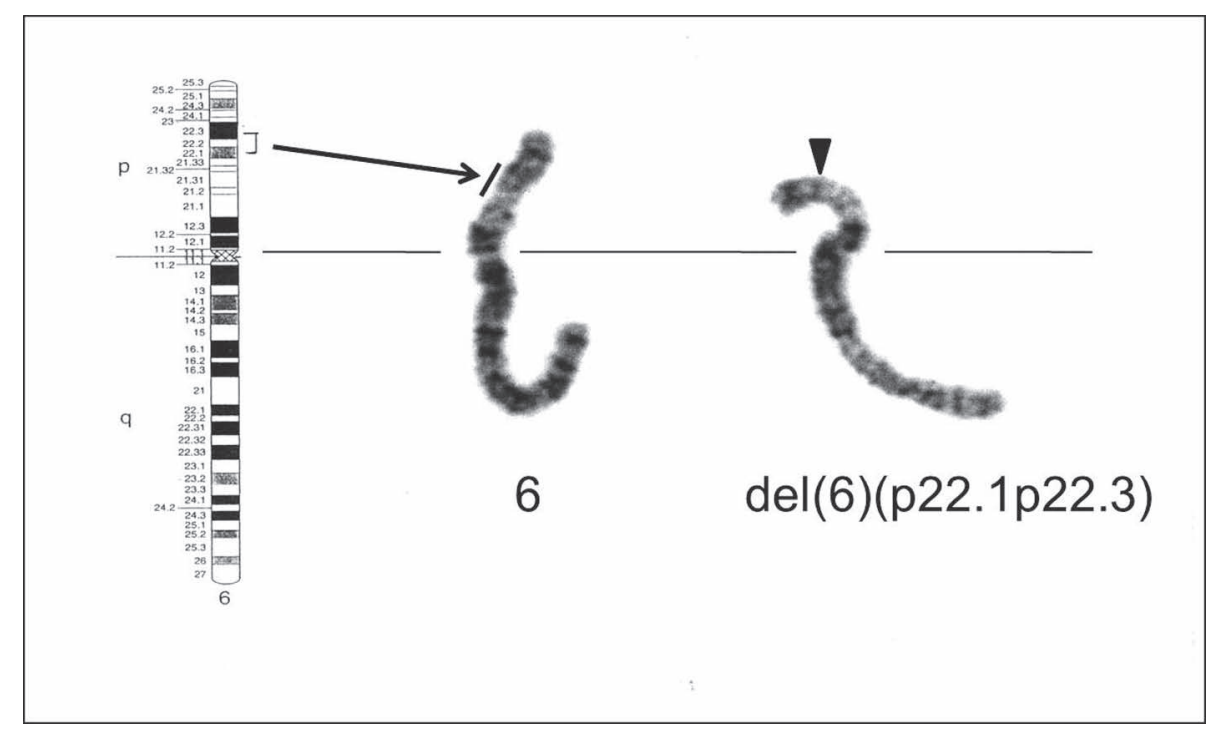

Fig. 2. Conventional chromosomal analyses of the patient.

Conventional chromosomal analyses disclosed interstitial deletion del(6)(p22.1p22.3). 


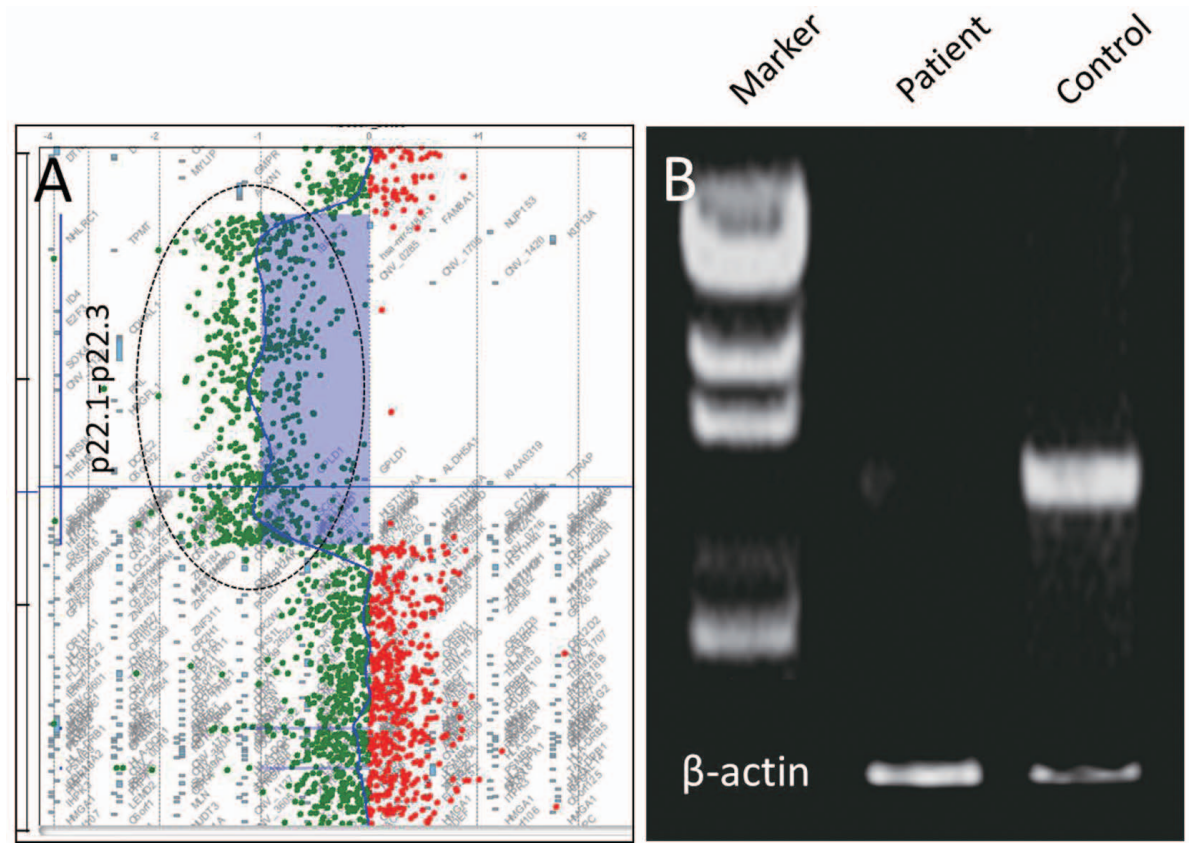

Fig. 3. CGH findings and PCR analyses for $E 2 F 3$ in the patient.

CGH disclosed nearly complete deletion of genes involving E2F3 localized on $6 \mathrm{p} 22.1$ to $6 \mathrm{p} 22.3$ (A), indicated by dotted circle. No band was detected for $E 2 F 3$ in contrast to the normal control (B).

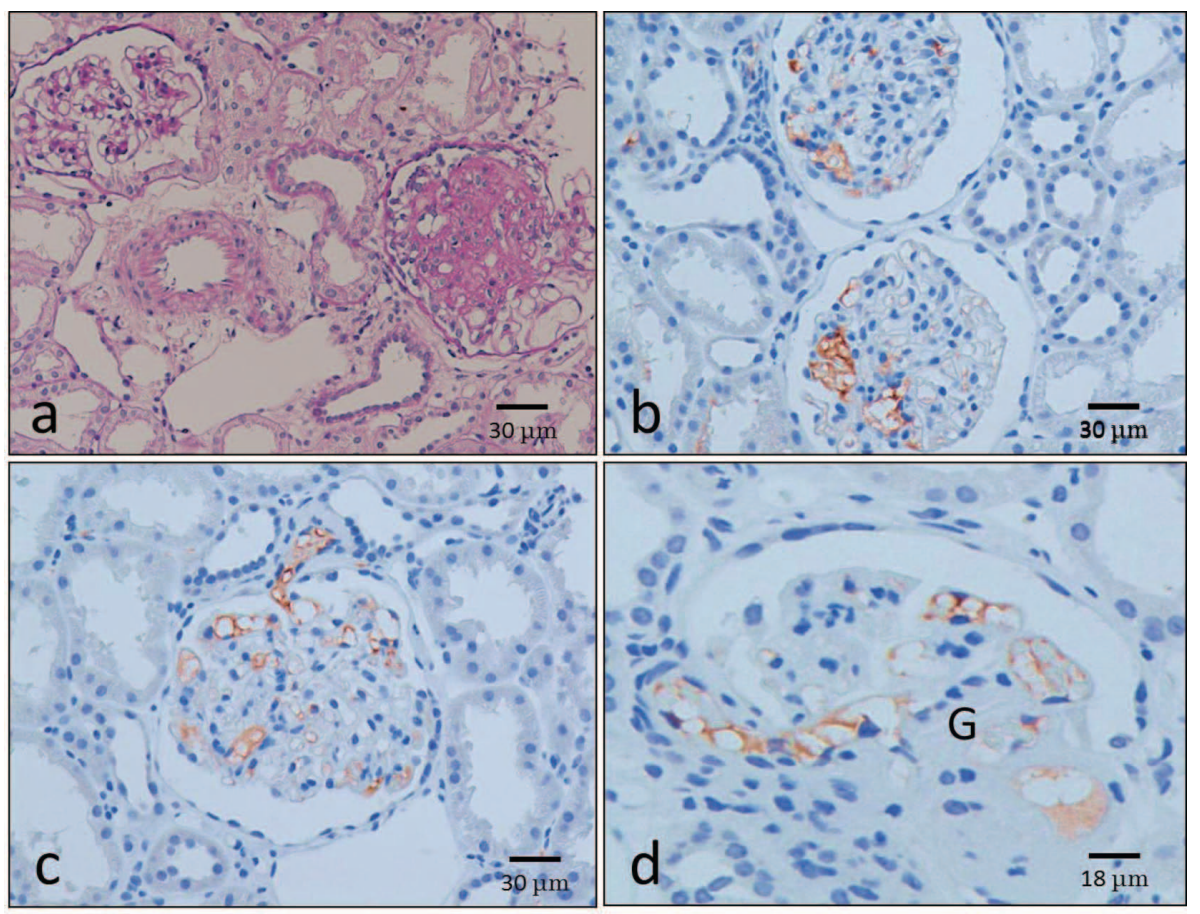

Fig. 4. Renal histologic findings and glomerular VEGF expression at the ages of 6 and 10 years.

At the age of 6 years, FSGS was detected (a); periodic acid-Schiff stain, $\times 200$. Excessive VEGF expression was observed mainly along glomerular endothelium, and less extensively in glomerular visceral epithelial cells (b). At the age of 10 years, intense VEGF expression persisted in glomeruli (c). VEGF expression also was evident in highly sclerotic glomeruli (d, indicated by $\mathrm{G})$. Immunoperoxidase staining; b and c, $\times 200$; $d, \times 400$.

family transcription factor modulates angiogenesis via p53-dependent transcriptional control of VEGF and also inducing apoptosis in the process of normal vascular morphologic development (Qin et al. 2006). E2F3 deletion also was detected employing a polymerase-chain reaction method (Fig. 3b), suggesting that glomerular architecture was compromised through defective glomerular angiogenesis. Accordingly, serum levels of VEGF and platelet- 
derived growth factor (PDGF) were measured, when the patients was 6 years old. The concentrations of VEGF were $177 \pm 21.4 \mathrm{pg} / \mathrm{mL}$ (upper limit of $33.3 \mathrm{pg} / \mathrm{mL}$ ), showing marked elevation, while the level of PDGF were $32.8 \mathrm{pg} /$ $\mathrm{mL}$ (upper limit, $50 \mathrm{pg} / \mathrm{mL}$ ). The similar results were obtained by repeated examinations. No abnormalities were found in the $W T 1$ and $\alpha$-actinin 4 genes.

Renal biopsy was performed, because when proteinuria gradually worsened from ages of 5; and FSGS was diagnosed from findings in the specimen (Fig. 4a). VEGF expression in renal tissue was investigated by immunohistologic staining. Glomeruli in the initial renal biopsy at 6 years of age showed marked expression along the endothelium of glomerular capillaries and also on some podocytes (Fig. 4b). This expression persisted at 10 years of age (Fig. 4c), with marked expression also present in glomeruli showing sclerotic lesions (Fig. 4d). In contrast, VEGF was only mildly expressed along the endothelium of the glomerular capillaries in normal control glomeruli (a kidney specimen resected because of renal trauma in a 10 -year-old child: data not shown).

The patient has been treated with combined therapy using an angiotensin-converting enzyme (ACE) inhibitor, an angiotensin-II receptor blockade (ARB), and spherules carbonaceous adsorbent, aiming to suppress renal deterioration of function. However, her renal function has worsened slowly over subsequent years. Laboratory data from the most recent check-up at age 10 were proteinuria, $0.5 \mathrm{~g} /$ day, blood urea nitrogen, $26 \mathrm{mg} / \mathrm{dL}$, serum creatinine, 1.52 , and creatinine clearance, $50.2 \mathrm{ml} / \mathrm{min} / 1.48 \mathrm{~m}^{2}$, suggesting moderate renal dysfunction.

\section{Discussion}

Glomerulogenesis depends on multiple growth factors functionally related to VEGF (Kitamoto et al. 1997). A variety of growth factors participate in maturation of the nephron, especially PDGF- $\beta$ and VEGF (Kitamoto et al. 1997, Lindahl et al. 1998). VEGF and its receptor, VEGFR2/Flk-1, also are important in regulating proliferation of glomerular cells and formation of glomerular capillary loops; differentiation of glomerular capillaries was suppressed experimentally by treatment with neutralizing antibodies for VEGF (Kitamoto et al. 1997). Additionally, VEGF and VEGF receptors are highly expressed in the kidney (Izzedine et al. 2007). VEGF is expressed in podocytes in the glomerulus, and VEGF receptors are present on endothelial, mesangial, and peritubular capillary cells. Therefore, signaling between endothelial cells and podocytes is essential for the proper development and maintenance of the filtration function of the kidney glomerulus. A recent study suggests that administration of VEGF antagonists to animal model of crescentic glomerulonephritis can ameliorate glomerular injuries and prevent advances of the renal functional deterioration (Kitamoto et al. 2008).

The transcription factor $E 2 F$ gene is implicated in cell division, apoptosis, and differentiation (Qin et al. 2006,
Chong et al. 2009, Martinez et al. 2010). Specific inactivation of $E 2 F 3$ in mouse embryo fibroblasts results in disruption of the centrosome duplication cycle (Saavedra et al. 2003 ). In addition, $E 2 F 3$ mutant mice typically die perinatally, while E2F3-deficient cells show a proliferation defect that correlates with impaired E2F target gene activation (Danielian et al. 2008). Since E2F3 is a known regulator of neural precursor proliferation (McClellan et al. 2007), our patient's mental developmental delay may have resulted from $E 2 F 3$ abnormality. Previous studies also have demonstrated that an $E 2 F$ family transcription factor including $E 2 F 3$ is essential for the process of angiogenesis through regulation of VEGF secretion and directing appropriate apoptosis (Qin et al. 2006). Mice deficient in a $E 2 F$ family transcription factor gene exhibit enhanced angiogenesis (Qin et al. 2006), while the proangiogenic phenotype in its deficiency results from VEGF overproduction and is prevented by VEGF blockade (Qin et al. 2006). Previous studies demonstrated that excessive expression of VEGF can cause unusual neovasculization in diabetic glomerulopathy and in glomeruli of VEGF-overexpressing transgenic rabbits (Kanesaki et al. 2005; Qin et al. 2006). Another animal study showed that mice overexpressing VEGF-A in podocytes die within 5 days (Liu et al. 2007); the glomerular tuft is collapsed, with most loops lacking endothelial cells, a consequence of a severe regulatory defect in glomerular angiogenesis. The present patient showed glomerular overexpression of VEGF accompanied by a markedly increased serum VEGF concentration. Glomerular VEGF deposition was evident in partially or extensively sclerotic glomeruli. These findings suggest a close relationship between development of FSGS and excessive VEGF expression caused by the $E 2 F 3$ deletion in our patient. On the other hand, intrarenal angiotensin II concentration increases according to the advancement of kidney sclerosis (Moeller 2010). Since angiotensin II stimulates synthesis of VEGF (Kang et al. 2006), the patient's VEGF-dependent renal injury could be further aggravated. Additionally since $E 2 F$ overexpression can trigger excessive apoptosis (Iaquinta et al. 2005), deletion of $E 2 F 3$ in our patient may preclude appropriate apoptosis needed to establish normal glomerular structure. In addition, increased level of VEGF in induced sputum has been demonstrated in patients with stable asthma, as well as during the exacerbation of disease (Gomulka and Liebhart 2009). The excessive synthesis of VEGF may cause patient's asthma.

Reported patients with this disease whose genetic abnormalities were complicated by renal or other urinary tract anomalies have shown hemizygous deletions in $6 \mathrm{p}$ similar to those in our patient. A kidney defect was observed in a patient with del(6)(p22.3-p24.3), and hydronephrosis caused by stenosis at the ureteropelvic junction developed in a patient with del(6)(p23p24.2) (Davies et al. 1999). In a patient with del(6)(p21.3), hydronephrosis affected the left kidney while the right kidney was aplastic (Suwanrath-Kengpol et al. 2004). Thus, similar partial 
deletions of $6 p$ are associated with heterogeneous clinical patterns for reasons not precisely known. However, there are no previous reports who described the renal histopathologic findings of patients with the partial deletion of chromosome $6 \mathrm{p}$. These renal disorders may result from the defect of $E 2 F 3$ mapped to $6 \mathrm{p} 22$.

FSGS can be caused by abnormalities of certain genes including $W T 1$ and $\alpha$-actinin 4 (D'Agati 2008). One may speculate that a gene or a combination of genes normally located on 6p may have contributed to occurrence of this condition. FSGS also is observed occasionally in some patients with mental retardation such as those with Galloway-Mowat syndrome (Sartelet et al. 2008). Thus, disease categories have been established in many patients, but the cause often is unclear. Among patients with idiopathic FSGS, an abnormality of $E 2 F$ family transcription factor genes involving $E 2 F 3$ may exist on chromosome 6p. Therefore, one might consider carrying out chromosomal analyses together with $\mathrm{CGH}$ in children with FSGS who also have mental retardation.

\section{Specific Remarks}

We have no conflicting interest for performing a present study. This manuscript has been seen and approved by all authors and that it is not under consideration for publication elsewhere in a similar form, in any language, except in abstract form. Genetic analyses and tissue staining for renal specimens were performed after we received approval from the Ethics Committee at Kinki University and obtained written informed consent from the patient's family members.

\section{Acknowledgments}

The study was partly supported by a Grant-in-Aid for Scientific Research from Morinaga Hoshikai to T.T. (from 2009 to 2010). We thank Naomi Jinno for assistance in manuscript preparation.

\section{References}

Boyer, O., Moulder, J.K. \& Somers, M.J. (2007) Focal and segmental glomerulosclerosis in children: a longitudinal assessment. Pediatr. Nephrol., 22, 1159-1166.

Chong, J.L., Tsai, S.Y., Sharma, N., Opavsky, R., Price, R., Wu, L., Fernandez, S.A. \& Leone, G. (2009) E2f3a and E2f3b contribute to the control of cell proliferation and mouse development. Mol. Cell. Biol., 29, 414-424.

D'Agati, V.D. (2008) The spectrum of focal segmental glomerulosclerosis: new insights. Curr. Opin. Nephrol. Hypertens., 17, 271-281.

Danielian, P.S., Friesenhahn, L.B., Faust, A.M., West, J.C., Caron, A.M., Bronson, R.T. \& Lees, J.A. (2008) E2f3a and E2f3b make overlapping but different contributions to total E2f3 activity. Oncogene, 27, 6561-6570.

Davies, A.F., Mirza, G., Sekhon, G., Turnpenny, P., Leroy, F., Speleman, F., Law, C., van Regemorter, N., Vamos, E., Flinter, F. \& Ragoussis, J. (1999) Delineation of two distinct 6p deletion syndromes. Hum. Genet., 104, 64-72.

Davies, A.F., Olavesen, M.G., Stephens, R.J., Davidson, R., Delneste, D., Van Regemorter, N., Vamos, E., Flinter, F., Abusaad, I. \& Ragoussis, J. (1996) A detailed investigation of two cases exhibiting characteristics of the $6 \mathrm{p}$ deletion syndrome. Hum. Genet., 98, 454-459.

Feber, A., Clark, J., Goodwin, G., Dodson, A.R., Smith, P.H., Fletcher, A., Edwards, S., Flohr, P., Falconer, A., Roe, T., Kovacs, G., Dennis, N., Fisher, C., Wooster, R., Huddart, R., Foster, C.S. \& Cooper, C.S. (2004) Amplification and overexpression of E2F3 in human bladder cancer. Oncogene, 23, $1627-1630$.

Gomułka, K. \& Liebhart, J. (2009) Vascular endothelial growth factor - structure, function and role in airways inflammation and the clinical course of asthma. Pneumonol. Alergol. Pol., 77, 549-553.

Iaquinta, P.J., Aslanian, A. \& Lees, J.A. (2005) Regulation of the Arf/p53 tumor surveillance network by E2F. Cold. Spring. Harb. Symp. Quant. Biol., 70, 309-316.

Izzedine, H., Rixe, O., Billemont, B., Baumelou, A. \& Deray, G. (2007) Angiogenesis inhibitor therapies: focus on kidney toxicity and hypertension. Am. J. Kidney Dis., 50, 203-218.

Izu, A., Yanagida, H., Sugimoto, K., Fujita, S., Okada, M. \& Takemura, T. (2011) Focal Segmental Glomerulosclerosis and Partial Deletion of Chromosome 6p: A Case Report. Clin. Nephrol. in press

Joh, K., Usui, N., Aizawa, S., Yamaguchi, Y., Chiba, S., Takahashi, T., Muramatsu, Y. \& Sakai, S. (1991) Focal segmental glomerulosclerosis associated with infantile spasms in five mentally retarded children: a morphological analysis on mesangiolysis. Am. J.Kidney Dis., 17, 569-577.

Kanesaki, Y., Suzuki, D., Uehara, G., Toyoda, M., Katoh, T., Sakai, H. \& Watanabe, T. (2005) Vascular endothelial growth factor gene expression is correlated with glomerular neovascularization in human diabetic nephropathy. J. Biol. Chem., 280, 21531-21538.

Kang, Y.S., Park, Y.G., Kim, B.K., Han, S.Y., Jee, Y.H., Han, K.H., Lee, M.H., Song, H.K., Cha, D.R., Kang, S.W. \& Han, D.S. (2006) Angiotensin II stimulates the synthesis of vascular endothelial growth factor through the p38 mitogen activated protein kinase pathway in cultured mouse podocytes. J. Mol. Endocrinol., 36, 377-388.

Kitamoto, Y., Taguma, Y., Arizono, K. \& Imamura, T. (2008) AntiVEGF therapy for crescentic glomerulonephritis. Am. J. Kidney Dis., 51, 710-711.

Kitamoto, Y., Tokunaga, H. \& Tomita, K. (1997) Vascular endothelial growth factor is an essential molecule for mouse kidney development. J. Clin. Invest., 99, 2351-2357.

Lindahl, P., Hellstrom, M., Kalen, M., Karlsson, L., Pekny, M., Pekna, M., Soriano, P. \& Betsholtz, C. (1998) Paracrine PDGF-B/PDGF-Rbeta signaling controls mesangial cell development in kidney glomeruli. Development, 125, 33133322.

Liu, E., Morimoto, M., Kitajima, S., Koike, T., Yu, Y., Shiiki, H., Nagata, M., Watanabe, T. \& Fan, J. (2007) Increased expression of vascular endothelial growth factor in kidney leads to progressive impairment of glomerular functions. J. Am. Soc. Nephrol., 18, 2094-2104.

McClellan, K.A., Ruzhynsky, V.A., Douda, D.N., Vanderluit, J.L., Ferguson, K.L., Chen, D., Bremner, R., Park, D.S., Leone, G. \& Slack, R.S. (2007) Unique requirement for Rb/E2F3 in neuronal migration: evidence for cell cycle-independent functions. Mol. Cell. Biol., 13, 4825-4843.

Martinez, L.A., Goluszko, E., Chen, H.Z., Leone, G., Post, S., Lozano, G., Chen, Z. \& Chauchereau, A. (2010) E2F3 is a mediator of DNA damage-induced apoptosis. Mol. Cell. Biol., 30, 524-536.

Moeller, M.J. (2010) Glomerular scarring: can we delay or even reverse glomerulosclerosis by RAAS inhibition? Nephrol. Dial. Transplant., 25, 2101-2103.

Palmer, C.G., Bader, P., Slovac, M.L., Comings, D.E. \& Pettenati, M.J. (1991) Partial deletion of chromosome 6p: delineation of the syndrome. Am. J. Med. Genet., 39, 155-160. 
Qin, G., Kishore, R., Dolan, C.M., Silver, M., Wecker, A., Luedemann, C.N., Thorne, T., Hanley, A., Curry, C., Heyd, L., Dinesh, D., Kearney, M., Martelli, F., Murayama, T., Goukassian, D.A., Zhu, Y. \& Losordo, D.W. (2006) Cell cycle regulator E2F1 modulates angiogenesis via p53-dependent transcriptional control of VEGF. Proc. Natl. Acad. Sci. USA, 103, 11015-11020.

Saavedra, H.I., Maiti, B., Timmers, C., Altura, R., Tokuyama, Y., Fukasawa, K. \& Leone, G. (2003) Inactivation of E2F3 results in centrosome amplification. Cancer Cell, 4, 333-346.

Sartelet, H., Pietrement, C., Noel, L.H., Sabouraud, P., Birembaut, P., Oligny, L.L., Roussel, B. \& Doco-Fenzy, M. (2008)
Collapsing glomerulopathy in Galloway-Mowat syndrome: a case report and review of the literature. Pathol. Res. Pract., 204, 401-406.

Suwanrath-Kengpol, C., Limprasert, P. \& Mitarnun, W. (2004) Prenatal diagnosis of deletion of chromosome $6 \mathrm{p}$ presenting with hydrops fetalis. Prenat. Diagn., 24, 887-889.

Takemura, Y., Koshimichi, M., Sugimoto, K., Yanagida, H., Fujita, S., Miyazawa, T., Miyazaki, K., Okada, M. \& Takemura, T. (2010) A tubulointerstitial nephritis antigen gene defect causes childhood-onset chronic renal failure. Pediatr. Nephrol., 25, 1349-1353. 